\title{
ON THE GENERALIZED RIEMANN INTEGRAL AND STOCHASTIC INTEGRAL
}

\author{
TACK-WANG LEE \\ (Received 10 May 1973; revised 17 March 1974) \\ Communicated by E. Strzelecki
}

\section{Introduction}

In Lee (submitted), the $G W$-integral (the generalized Riemann integral using Wiener measure) is defined. The object of this article is to define stochastic integral in the set up given in Lee (submitted). We also investigate the connection between the stochastic integral defined with the Legesgue counter part, the PaleyWiener-Zygmund integral in Paley, Weiner and Zygmund (1933). Applications of the stochastic integral will be explained elsewhere.

\section{GWS-Integral}

Notations given in Henstock $(1963,1967,1968)$ and Lee (submitted) will be used freely without further explanations.

Let $f(t)$ be a real function defined on $[a, b)$. We put

$$
\mathbf{S}(\delta ; f)=\{(\mathscr{D}) \Sigma f(t)(v-u)\}
$$

where $\mathscr{D}$ is a division of $[a, b)$ compatible with the function $\delta>0$ defined on $[a, b]$. Suppose $f$ is (generalized Riemann) integrable with respect to the usual interval function on $[a, b)$. Then it can be shown that

Let

$$
\bigcap_{\delta>0} \mathbf{S}(\delta ; f)=\int_{a}^{b} f d t
$$

$$
S^{*}\left(\mathscr{C}_{o} ; \delta ; f\right)=\{(\mathscr{D}) \Sigma f(t)(x(v)-x(u))
$$

where $\mathscr{D}$ is a division of $[a, b)$ compatible with $\delta>0$ and $x(t) \in \mathscr{C}_{0}$. Recall that $\mathscr{C}_{o}$ is the set of all continuous functions defined on $[a, b]$ and $x(a)=0$. Obviously, $\mathbf{S}^{*}\left(\mathscr{C}_{o} ; \delta ; f\right)$ is a set of functionals defined on $\mathscr{C}_{0}$.

Suppose that there exists some functional $\mathbf{k}[x]$ defined on $\mathscr{C}_{0}$ with the property that, given $\varepsilon>0$ there exists $\delta>0$ such that 


$$
\text { (G) } \int_{\epsilon_{0}}|\mathbf{s}-\mathbf{k}[x]|^{2} d W_{\sigma}<\varepsilon
$$

for all $\mathbf{s} \in \mathbf{S}^{*}\left(\mathscr{C}_{0} ; \delta ; f\right)$. Then we say that $\mathbf{k}[x]$ is the stochastic integral of $f$ with respect to $x(t) \in \mathscr{C}_{0}$ for the generalized Riemann integral using Wiener measure, in short $G W S$-integral. It is not difficult to see that $\mathbf{k}[x]$ is unique almost everywhere if it exists. Indeed, if $\mathbf{k}^{*}[x]$ is another such functional satisfying (1) with $\delta$ replaced by $\delta^{*}$, then we have

$$
\begin{gathered}
\left((G) \int_{\mathscr{\ell}_{0}}\left|\mathbf{k}[x]-\mathbf{k}^{*}[x]\right|^{2} d W_{\sigma}\right)^{\frac{1}{2}} \leqq\left((G) \int_{\mathscr{\ell}_{0}}|\mathbf{k}[x]-\mathrm{s}[x]|^{2} d W_{\sigma}\right)^{\frac{1}{2}} \\
+\left((G) \int_{\mathscr{C}_{\sigma}}\left|\mathrm{s}[x]-\mathbf{k}^{*}[x]\right|^{2} d W_{\sigma}\right)^{\frac{1}{2}}<\varepsilon^{\frac{1}{2}}+\varepsilon^{\frac{1}{2}}=2 \varepsilon^{\frac{1}{2}}
\end{gathered}
$$

for all $\mathbf{s} \in \mathbf{S}^{*}\left(\mathscr{C}_{0} ; \delta^{\prime} ; f\right)$ where $\delta^{\prime}=\min \left(\delta, \delta^{*}\right)$. That is

Hence we write

$$
\text { (G) } \int_{\mathscr{C}_{0}}\left|\mathbf{k}[x]-\mathbf{k}^{*}[x]\right|^{2} d W_{\sigma}=0 .
$$

$$
\mathbf{k}[x]=(G W S) \int_{a}^{b} f d x(t) .
$$

Remarks. (i) (GWS) $\int_{a}^{b} f d x(t)$ is not the same as the usual $\int_{a}^{b} f d x(t)$ for each $x \in \mathscr{C}_{c}$. But, if $f$ is of bounded variation in $[a, b]$, then $\int_{a}^{b} f d x(t)$ $=(G W S) \int_{a}^{b} f d x(t)$ as we shall see.

(ii) Other norms in (1) may also be used. In this article we use only this norm. For other norms, we explain in a subsequent article.

Before going further, we introduce further notations. Let $\mathscr{D}$ be a division of $[a, b)$ and $(I, t)$ be an interval-point pair from $\mathscr{D}$. We define $\phi(\mathscr{D})$ to be the step function such that for all $t^{\prime} \in I, \phi\left(t^{\prime}\right)=f(t)$. If $\mathscr{D}$ and $\mathscr{D}^{*}$ are two such divisions, then we write $\mathscr{D} \vee \mathscr{D}^{*}$ to be the division of $[a, b)$ which includes all the division points from $\mathscr{D}$ and $\mathscr{D}^{*}$. Note that $\mathscr{D} \vee \mathscr{D}^{*}$ is not necessarily compatible with $\delta$ in the usual sense. Then we define $\left(\phi-\phi^{*}\right)\left(\mathscr{D} \vee \mathscr{D}^{*}\right)$ to be the step function such that for each $I^{*}$ from $\mathscr{D} \vee \mathscr{D}^{*},\left(\phi-\phi^{*}\right)\left(t^{*}\right)=\phi\left(t^{*}\right)-\phi^{*}\left(t^{*}\right)$ for all $t^{*} \in I^{*}$ from $\mathscr{D} \vee \mathscr{D}^{*}$ and $\phi\left(t^{*}\right)-\phi^{*}\left(t^{*}\right)=$ constant in $I^{*}$.

Lemma. Let $t_{i}(i=0,1, \cdots, n)$ be the division points of $\mathscr{T}$. If

$$
F[x]=(\mathscr{D}) \sum c_{i}\left(x_{i}-x_{i-1}\right)
$$

where $c_{i}$ are arbitrary fixed values and $x_{i}=x\left(t_{i}\right), i=0,1, \cdots, n$, then $F[x]$ is $G W$-integrable (see Lee (submitted)) and

$$
\text { (G) } \int_{\varnothing}(F[x])^{2} d W_{\sigma}=\sigma^{2}(\mathscr{D}) \Sigma c_{i}^{2}\left(t_{i}-t_{i-1}\right)
$$


Proof. Using the Lemma 4.2 in Lee (submitted), we can easily show that $F[x]$ is $G W$-integrable. Hence we need only to show the equality.

By the New Fubini's Theorem (Theorem 4.1 in Lee (submitted)), we have

(G) $\int_{\mathscr{\ell}_{0}}(F[x])^{2} d W_{s}=\left(\sigma^{2} 2 \pi\right)^{-n \mid 2}\left(\prod_{i=1}^{n}\left(t_{i}-t_{i+1}\right)\right)^{-\frac{1}{2}}$

$$
\times \int_{R^{n}}\left((\mathscr{D}) \sum c_{i}\left(x_{i}-x_{i-1}\right)\right)^{2} \exp \left(-\left(2 \sigma^{2}\right)^{-1} \sum_{i=1}^{n} \frac{\left(x_{i}-x_{i-1}\right)^{2}}{t_{i}-t_{i-1}}\right) d x_{1} \cdots d x_{n} .
$$

In succession, we put $x_{i}=x_{i-1}+y_{i}\left(t_{i}-t_{i-1}\right)^{\frac{1}{2}}(i=1,2, \cdots, n)$. Then the right hand side of (2) is equal to

$$
\begin{aligned}
&\left(\sigma^{2} 2 \pi\right)^{-n \mid 2}\left(\prod_{i=1}^{n}\left(t_{i}-t_{i-1}\right)\right)^{-\frac{1}{2}} \int_{k^{n}}\left(\sum_{i=1}^{n} c_{i}^{2} y_{i}^{2}\left(t_{i}-t_{i-1}\right)\right) \\
& \times \exp \left(-\left(2 \sigma^{2}\right)^{-1} \sum_{i=1}^{n} y_{i}^{2}\right) d y_{1} \cdots d y_{n} \\
&=\left(\sigma^{2} 2 \pi\right)^{-\frac{1}{2}} \sum_{i=1}^{n} c_{i}^{2}\left(t_{i}-t_{i-1}\right) \int_{-\infty}^{\infty} y^{2} \exp \left(-\left(2 \sigma^{2}\right)^{-1} y^{2}\right) d y=\sigma^{2} \sum_{i=1}^{n} c_{i}^{2}\left(t_{i}-t_{i-1}\right)
\end{aligned}
$$

using the fact that

$$
\begin{aligned}
& \int_{-\infty}^{\infty} \int_{-\infty}^{\infty}\left(x_{i}-x_{i-1}\right)\left(x_{i-1}-x_{i-2}\right) \exp \left(-\left(2 \sigma^{2}\right)^{-1}\left(\left(x_{i}-x_{i-1}\right)^{2}\right.\right. \\
& \left.+\left(x_{i-1}-x_{i-2}\right)^{2}\right) d x_{i} d x_{i-1}=0, \quad i=2,3, \cdots, n+1 .
\end{aligned}
$$

By the Lemma, we have the following result for $G W S$-integral:

Proposition. Let $f \in L^{2}([a, b])$. Then (GWS) $\int_{a}^{b} f d x(t)$ exists except in $a$ set of $W_{\sigma}$-variation zero and

$$
\sigma^{2}\|f\|_{2}^{2}=(G) \int_{\mathscr{G}_{o}}\left((G W S) \int_{a}^{b} f d x(t)\right)^{2} d W_{\sigma}
$$

where $\|\cdot\|_{2}$ is the $L^{2}([a, b])$-norm.

Proof. By the hypothesis that $f \in L^{2}([a, b])$, we have given $\varepsilon>0$, there exists $\delta>0$ such that

$$
\int_{a}^{b}(\phi(\mathscr{D})-f)^{2} d t<\varepsilon
$$

for each $\phi(\mathscr{D})$ and $\mathscr{D}$ is a division of $[a, b)$ compatible with $\delta>0$. (See Theorem 47.2 in Henstock (1967)). Hence for any two simple (step) functions $\phi(\mathscr{D})$ and $\phi^{*}\left(\mathscr{D}^{*}\right)$ where $\mathscr{D}$ and $\mathscr{D}^{*}$ are compatible with $\delta$, we have 
That is

$$
\begin{aligned}
\mathscr{Q}^{\frac{1}{2}} & =\left(\left(\mathscr{D} \vee \mathscr{C}^{*}\right) \Sigma\left(\left(\phi-\phi^{*}\right)\left(t^{*}\right)\right)^{2}(v-u)\right)^{\frac{1}{2}}=\left(\int_{a}^{b}\left(\phi-\phi^{*}\right)^{2} d t\right)^{\frac{1}{2}} \\
& \leqq\left(\int_{a}^{b}(\phi-f)^{2} d t\right)^{\frac{1}{2}}+\left(\int_{a}^{b}\left(\phi^{*}-f\right)^{2} d t\right)^{\frac{1}{2}}<\varepsilon^{\frac{1}{2}}+\varepsilon^{\frac{1}{2}}=2 \varepsilon^{\frac{1}{2}}
\end{aligned}
$$

$$
\mathscr{2}=\left(\mathscr{D} \vee \mathscr{D}^{*}\right) \sum\left(\phi-\phi^{*}\right)^{2}(v-u)<4 \varepsilon .
$$

Define $\mathbf{s}(\mathscr{D})=(\mathscr{D}) \sum f(t)(x(v)-x(u))$. Now we observe that for the corresponding $\mathbf{s}(\mathscr{D}), \mathbf{s}^{*}(\mathscr{D}) \in \mathbf{S}^{*}\left(\mathscr{C}_{o} ; \delta ; f\right)$, the functional $\mathbf{s}(\mathscr{D})-\mathbf{s}\left(\mathscr{D}^{*}\right)$ depends on the division points of $\mathscr{D} \vee \mathscr{D}^{*}$ only. Therefore by the Lemma, (3) and the New Fubini's Theorem, we have

$$
\text { (G) } \begin{aligned}
&\left(\mathrm{s}(\mathscr{D})-\mathrm{s}^{*}\left(\mathscr{Y}^{*}\right)\right)^{2} d W_{\sigma} \\
&=\left(\sigma^{2} 2 \pi\right)^{-n \mid 2}\left(\prod_{i=1}^{n}\left(t_{i}-t_{i-1}\right)\right)^{-\frac{1}{2}} \int_{R^{n}}\left(\left(\mathscr{D} \vee \mathscr{D}^{*}\right) \sum\left(\left(\phi-\phi^{*}\right)\left(t^{*}\right)(x(v)-x(u))\right)\right)^{2} \\
& \times \exp \left(-\left(2 \sigma^{2}\right)^{-1} \sum_{i=1}^{n} \frac{\left(x_{i}-x_{i-1}\right)^{2}}{t_{i}-t_{i-1}} d x_{1} \cdots d x_{n}\right. \\
&=\left(\sigma^{2} 2 \pi \mathscr{Z}\right)^{-\frac{1}{2}} \int_{-\infty}^{\infty} y^{2} \exp \left(-\nu^{2} /\left(2 \sigma^{2} \mathscr{Z}\right)\right) d y=\sigma^{2} \mathscr{Z}<4 \sigma^{2} \varepsilon
\end{aligned}
$$

where $x_{i}=x\left(t_{i}\right), x(a)=x\left(t_{0}\right)=0, x(b)=x\left(t_{n}\right)(i=1,2, \cdots, n)$ for some $n\left(\mathscr{D} \vee \mathscr{D}^{*}\right)$.

Setting $\varepsilon_{m}=\varepsilon 2^{-m}(m=1,2, \cdots)$, and $\delta_{m}>0$ corresponding to $\varepsilon_{m}$ in (3), we observe that $\left\{\mathbf{s}\left(\mathscr{D}_{m}\right)\right\}$ where $\mathbf{s}\left(\mathscr{D}_{m}\right) \in \mathbf{S}^{*}\left(\mathscr{C}_{o} ; \delta_{m} ; f\right)$ is a Cauchy sequence in $\mathscr{L}^{2}\left(\mathscr{C}_{o}\right)$-norm. Hence by the completeness of $\mathscr{L}^{2}\left(\mathscr{C}_{o}\right)$, we conclude that there exists a functional $\mathbf{k}[x]$ defined on $\mathscr{C}_{o}$ such that

$$
\lim _{m \rightarrow \infty} \mathbf{s}\left(\mathscr{D}_{m}\right)=\mathbf{k}[x]
$$

in $\mathscr{L}^{2}\left(\mathscr{G}_{o}\right)$. Take $\mathscr{D}$ arbitary and compatible with $\delta$, and $\mathscr{D}^{*}$ as $\mathscr{T}_{m}$ in (3). Then by the Lemma, Fatou's lemma, and (3)

$$
\begin{aligned}
\mathbf{0} & \leqq(G) \int_{\mathscr{C}_{0}}(\mathbf{s}(\mathscr{D})-\mathbf{k}[x])^{2} d W_{\sigma} \leqq \liminf _{m \rightarrow \infty}(G) \int_{\mathscr{C}_{0}}\left(\mathbf{s}(\mathscr{D})-\mathbf{s}\left(\mathscr{D}_{m}\right)\right)^{2} d W_{\sigma} \\
& =\liminf _{m \rightarrow \infty}\left(\mathscr{D} \vee \mathscr{D}_{m}\right) \sum\left(\phi(\mathscr{O})-\phi\left(\mathscr{O}_{m}\right)\right)^{2}(t)(v-u) \leqq 4 \varepsilon .
\end{aligned}
$$

It follows that

$$
\lim _{m \rightarrow \infty} \mathbf{s}\left(\mathscr{D}_{m}\right)=\mathbf{k}[x]=(G W S) \int_{a}^{b} f d x(t)
$$

in $\mathscr{L}^{2}\left(\mathscr{C}_{0}\right)$. 
Now, using the Lemma and the New Fubini's Theorem, we have

$$
\text { (G) } \int_{\mathscr{C}_{0}}\left(\mathbf{s}\left(\mathscr{D}_{m}\right)\right)^{2} d W_{\sigma}=\phi^{2}\left(\mathscr{D}_{m}\right) \Sigma \phi_{m}^{2}(t)(v-u)
$$

where $\phi_{m}=\phi\left(\mathscr{D}_{m}\right)$. Further, we observe that

$$
\text { (G) } \int_{\mathscr{C}_{0}}\left(\mathbf{k}[x]-\mathbf{s}\left(\mathscr{D}_{m}\right)\right)^{2} d W_{\sigma} \text { and }(G) \int_{\mathscr{C}_{0}}\left|\mathbf{k}[x]\left(\mathbf{k}[x]-\mathbf{s}\left(\mathscr{D}_{m}\right)\right)\right| d W_{\sigma}
$$

tend to 0 as $m \rightarrow \infty$ by the given condition of $\mathbf{s}\left(\mathscr{D}_{m}\right)$ and Cauchy-Schwarz inequality. Also, we obtain the equality

$$
\begin{aligned}
\lim _{m \rightarrow \infty}(G) \int_{\mathscr{C}_{0}}\left(\mathbf{k}[x]-\mathbf{s}\left(\mathscr{D}_{m}\right)\right)^{2} d W_{\sigma} \\
=-(G) \int_{\mathscr{C}_{0}}(\mathbf{k}[x])^{2} d W_{\sigma}+2 \lim _{m \rightarrow \infty}(G) \int_{\mathscr{C}_{\bullet}} \mathbf{k}[x]\left(\mathbf{k}[x]-\mathbf{s}\left(\mathscr{D}_{m}\right)\right) d W_{\sigma} \\
\quad+\lim _{m \rightarrow \infty}(G) \int_{\mathscr{C}_{0}}\left(\mathbf{s}\left(\mathscr{D}_{m}\right)\right)^{2} d W_{\sigma}
\end{aligned}
$$

by the identity $(a-b)^{2}=-a^{2}+2 a(a-b)+b^{2}$. Consequently

$$
\begin{aligned}
& \text { (G) } \int_{\mathscr{C}_{n}} \mathbf{k}^{2}[x] d W_{\sigma}=\lim _{m \rightarrow \infty}(G) \int_{\mathscr{C}_{0}}\left(\mathbf{s}\left(\mathscr{D}_{m}\right)\right)^{2} d W_{\sigma}=\sigma^{2} \lim _{m \rightarrow \infty}\left(\mathscr{D}_{m}\right) \sum \phi_{m}^{2}(v-u) \\
& =\sigma^{2}\|f\|_{2}^{2} \\
& \text { as } \\
& \lim _{m \rightarrow \infty} \int_{a}^{b}\left|\phi_{m}\right|(t)-\left.f(t)\right|^{2} d t=0 .
\end{aligned}
$$

This completes the proof.

Remark. Note that the linear mapping $f \rightarrow(G W S) \int_{a}^{b} f d x(t)$ can be seen as 'nearly' isometry embedding of $L^{2}([a, b])$ into $\mathscr{L}^{2}\left(\mathscr{C}_{o}\right)$. This is rather important as we shall see in subsequent articles.

COROLlaRY. Let $f$ be a function of bounded variation on $[a, b]$. Then (GWS) $\int_{a}^{b} \stackrel{b}{f} d x(t)$ exists and

$$
(G W S) \int_{a}^{b} f d x(t)=\int_{a}^{b} f d x(t)
$$

where the integral on the right hand side is the generalized Riemann integral.

Proof. By the Proposition, $(G W S) \int_{a}^{b} f d x(t)$ exists as $f \cdot \in L^{2}([a, b])$. Further $\mathbf{I}=\int_{a}^{b} f d x(t)$ exists as the generalized Riemann integral and the Riemann- 
Stieltjes integral since $f$ is of bounded variation. Besides, their values are equal. (See Henstock (1967)). Hence

$$
\mathbf{I}=(G R) \int_{a}^{b} f d x(t)=(R S) \int_{a}^{b} f d x(t)=\lim _{m \rightarrow \infty} \mathbf{s}\left(\mathscr{D}_{m}\right)
$$

where $\mathscr{D}_{m}(m=1,2, \cdots)$ has the same meaning as in the proof of the Proposition while $(G R)$ and $(R S)$ denote the integrals exist in the generalized Riemann and Riemann-Stieltjes senses respectively. Hence we have

$$
\mathbf{I}=(G R) \int_{a}^{b} f d x(t)=(G W S) \int_{a}^{b} f d x(t) .
$$

\section{GWS-Integral and Paley-Wiener-Zygmund Integral}

First of all, we recall the definition of Paley-Wiener-Zygmund integral, written as $P W Z$-integral. Let $\left\{\psi_{k}(t)\right\}$ be an orthonormal basis of $L^{2}([a, b])$ and each of $\psi_{k}(t)$ be of bounded variation in $[a, b]$. Furthermore, let $\Sigma_{k=0}^{\infty} a_{k} \psi_{k}$ be the Fourier expansion of $f$ in $L^{2}([a, b])$. Then the PWZ-integral is given by

$$
(P W Z) \int_{a}^{b} f d x(t)=\lim _{n \rightarrow \infty}(L) \int_{a}^{b}\left(\sum_{k=0}^{n} a_{k} \psi_{k}(t)\right) d x(t)
$$

except in a set of Wiener measure zero where $(P W Z)$ and $L)$ mean the integrals exist in PWZ and Lebesgue senses respectively.

We are now in the position to prove the following result which links up the $G W S$-integral with $P W Z$-integral.

ThEOREM. Let $f,\left\{\psi_{k}(t)\right\}$ be given as above. Suppose $\Sigma_{k=0}^{\infty} a_{k} \psi_{k}(t)$ is the Fourier expansion of $f$ in $L^{2}([a, b])$. Then

$$
\begin{aligned}
(G W S) & \int_{a}^{b} f d x(t)=\lim _{n \rightarrow \infty}(G W S) \int_{a}^{b}\left(\sum_{k=0}^{n} a_{k} \psi_{k}(t)\right) d x(t) \\
= & \lim _{n \rightarrow \infty}(G R) \int_{a}^{b}\left(\sum_{k=0}^{n} a_{k} \psi_{k}(t)\right) d x(t)=\lim _{n \rightarrow \infty}(L) \int_{a}^{b}\left(\sum_{k=0} a_{k} \psi_{k}(t)\right) d x(t) \\
= & (P W Z) \int_{a}^{b} f d x(t)
\end{aligned}
$$

except in a set of $W_{\sigma}$-variation zero.

Proof. Using the ideas as in the proof of the Proposition, we have

$$
\text { (G) } \int_{\mathscr{B}_{a}}\left(\mathbf{s}(\mathscr{D})-(G W S) \int_{a}^{b} f d x(t)\right)^{2} d W_{a}<\varepsilon
$$

for all $\mathbf{s}(\mathscr{D}) \in \mathbf{S}^{*}\left(\mathscr{C}_{0} ; \delta ; f\right)$, 


$$
(G) \int_{\mathscr{C}_{0}}\left(\mathbf{s}^{*}\left(\mathscr{V}^{*}\right)-(G W S) \int_{a}^{b}\left(\sum_{k=0}^{m} a_{k} \psi_{k}(t)\right) d x(t)\right)^{2} d W_{\sigma}<\varepsilon
$$

for a fixed $m$ which may depend on $\varepsilon$, for all $\mathbf{s}^{*}\left(\mathscr{D}^{*}\right) \in \mathbf{S}^{*}\left(\mathscr{C}_{o} ; \delta ; \sum_{k=0}^{m} a_{k} \psi_{k}(t)\right)$,

$$
\begin{aligned}
& \left.\int_{a}^{b}\left(\sum_{k=0}^{m} a_{k} \psi_{k}(t)-f(t)\right)\right)^{2} d t<\varepsilon, \\
& \int_{a}^{b}\left(\phi_{\Re}(t)-f(t)\right)^{2} d t<\varepsilon,
\end{aligned}
$$

and

$$
\int_{a}^{b}\left(\phi_{y *}(t)-\sum_{k=0}^{m} a_{k} \psi_{k}(t)\right)^{2} d t<\varepsilon
$$

by taking $\delta$ small enough and $m$ large enough, where $\phi_{\mathscr{O}}$ and $\phi_{\mathscr{P}}$. denote the simple functions $\phi(\mathscr{D})$ and $\phi\left(\mathscr{D}^{*}\right)$ respectively.

Combining (4), (5), (6), (7) and (8) we have

$$
\begin{aligned}
& N^{\frac{1}{2}}=\left((G) \int_{\mathscr{C}_{o}}\left((G W S) \int_{a}^{b} f d x(t)-(G W S) \int_{a}^{b}\left(\sum_{k=0}^{m} a_{k} \psi_{k} d x(t)\right)^{2} d W_{v}\right)^{\frac{1}{2}}\right. \\
\leqq & \left((G) \int_{\mathscr{C}_{0}}\left((G W S) \int_{a}^{b} f d x(t)-\mathbf{s}(\mathscr{D})\right)^{2} d W_{\sigma}\right)^{\frac{1}{2}}+\left((G) \int_{\mathscr{C}_{a}}\left(\mathbf{s}(\mathscr{D})-\mathbf{s}^{*}\left(\mathscr{D}^{*}\right)\right)^{2} d W_{\sigma}\right)^{\frac{1}{2}} \\
+ & \left((G) \int_{\mathscr{C}_{n}}\left((G W S) \int_{a}^{b}\left(\sum_{k=0}^{m} a_{k} \psi_{k}\right) d x(t)-\mathbf{s}^{*}\left(\mathscr{D}^{*}\right)\right)^{2} d W_{\sigma}\right)^{\frac{1}{2}}<\varepsilon^{\frac{1}{2}}+3 \varepsilon^{\frac{1}{2}}+\varepsilon^{\frac{1}{2}}=5 \varepsilon^{\frac{1}{2}}
\end{aligned}
$$

since, by the Lemma

$$
\begin{aligned}
& \left((G) \int_{G_{0}}\left(\mathbf{s}(\mathscr{D})-\mathbf{s}^{*}\left(\mathscr{D}^{*}\right)\right)^{2} d W_{\sigma}\right)^{\frac{1}{2}}=\left(\int_{a}^{b}\left(\phi_{\mathscr{B}}(t)-\phi_{\mathscr{P}}(t)\right)^{2} d t\right)^{\frac{1}{2}} \\
& \leqq\left(\int_{a}^{b}\left(\left(\phi_{\mathscr{P}}(t)-f(t)\right)^{2} d t\right)^{\frac{1}{2}}+\left(\int_{a}^{b}\left(\phi_{\mathscr{D}}(t)-\sum_{-k=0}^{m} a_{k} \psi_{k}(t)\right)^{2} d t\right)^{\frac{1}{2}}\right. \\
& +\left(\int_{a}^{b}\left(f(t)-\sum_{k=0}^{m} a_{k} \psi_{k}(t)\right)^{2} d t\right)^{t} \\
& <\varepsilon^{\frac{1}{2}}+\varepsilon^{\frac{1}{2}}+\varepsilon^{\frac{1}{2}}=38^{\frac{1}{2}} \text {. }
\end{aligned}
$$

Hence

$$
\mathbf{N}<25 \varepsilon
$$

for all sufficiently large $m$. Therefore

$$
\text { (G) } \int_{\mathscr{C}_{o}}\left|(G W S) \int_{a}^{b} f d x(t)-(G W S) \int_{a}^{b}\left(\sum_{k=0}^{m} a_{k} \psi_{k}(t)\right) d x(t)\right| d W_{\sigma}<258
$$

for all sufficiently large $m$ as $(G) \int_{\epsilon_{0}} d W_{\sigma}=1$. The assertion now follows readily. 


\section{Conclusions}

The GWS-integral is in fact related to the so-called stochastic integral in Probability Theory. However, the kind of stochastic integrals we have discussed is merely the simplest kind. For the more general stochastic integrals, we shall explain the theory in subsequent articles. At the moment, the author has applied the theory to study the so-called Ito-belated integral given by McShane (to appear). The theory here is also generalized to more general division spaces.

The $G W$-integral and $G W S$-integral can also be used to simplify many proofs of Wiener integrals. We shall publish these works elsewhere.

\section{Acknowledgements}

The author wishes to express his gratitudes to the referee for some valuable suggestions, removing some errors and in particular, the proof of the equality in the Corollary.

\section{References}

R. Henstock (1963), Theory of Integration (Butterworth, London, 1963).

R. Henstock (1967), Linear Analysis, (Butterworth, London, 1967).

R. Henstock (1968), 'A Riemann-Type Integral of Lebesgue Power', Canad. J. Math. 20 78-87.

Tack-Wang Lee (submitted), 'On A New Approach To Functional Integration Theory', Proc. Lond. Math. Soc.

Tack-Wang Lee, Ph. D. Thesis, (Lancaster University, 1970.)

E. J. McShane (to appear), 'Stochastic Integration Theory'.

R. E. A. C. Paley, N. Wiener \& A. Zygmund (1933), 'Notes On Random Functions', Math. Z. 37, 647-688.

Department of Mathematics

University of Ghana

Legon, Ghana.

Present Address:

The School of Arts and Science

Tunku Abdul Rahman College

P.O. Box 979, Kuala Lumpur, Malaysia. 